\title{
Research on Aesthetic Training of Martial Arts Routines in College Physical Education
}

\author{
Huang Long \\ Institute of Physical Education \\ Chongqing Technology and Business University \\ Chongqing, China \\ 252963676@qq.com
}

\begin{abstract}
As a traditional Chinese culture with enduring charm, different cultural functions of martial arts have developed aesthetic feeling with traditional Chinese characteristics in the perspective of aesthetics research through the implementation of college teaching methods. Using the methods of literature and logic induction, this paper studies the recessive aesthetic cultivation of strength beauty, shape beauty and culture beauty in martial art education from three aspects: combination of instruction with practice, body pattern and culture appeal.
\end{abstract}

Keywords-martial arts, colleges and universities, physical education, aesthetic

\section{INTRODUCTION}

The physical form of martial arts has covered the understanding of the meaning of Chinese traditional culture. From the appearance, the formation and development of martial arts are always around the original essence of "fight" and "kill". In college teaching, based on the performance characteristics of martial arts' primitive essence, the aesthetic angle of martial arts can't be equated with the direct access of music or painting. Instead, it "uses body movement, performance form and method variation to make the trainer himself and others have some aesthetic pleasure"[1]. Based on this, the aesthetic of martial arts is the understanding of meaning implied in the martial arts actions, and the recessive aesthetic habits acquired through the practice of martial arts actions.

\section{Cultural Fit of Martial Art Teaching And College PHYSICAL EDUCATION}

The physical form of martial arts, in the cultural level, is some "body technique" that the members of our nation show through their understanding of their own culture[2]. Then, the body technique of martial arts can be understood as physical movements shown under the original state of martial arts through the understanding of martial arts culture. Martial arts' physical movement form is also called moves. "Move" means attack and defense in martial arts and intuitive forms shown by the body. Physical techniques in college education and martial arts, in the perspective of function, are able to meet the purpose of body building and exercise through body movements.
College physical education does not mean letting students simply master the sports techniques. It means that take advantage of different forms of exercise to "impact students, so as to enhance students' physical fitness and develop their physical literacy."[3] The impact of sports on teaching subject is manifold, and martial arts, as a part of college sports and an intuitive and specific martial arts teaching means, has the traditional Chinese sports impact. The external physical performance of martial arts emphasizes the control of body movements in the process; the intrinsic martial virtue cultivation highlights the psychological self-control and adaptation of personality and morality.

\section{TEACHING Forms OF COLlege MARTIAL ART Routines}

College martial arts teaching is an organic combination of martial arts culture and school physical education. On the basis of fully retaining the unique characteristics of martial arts, let students master technique essentials, understand the connotation of martial arts and enhance cultural identity through teaching methods of physical education. The teaching forms, in the practice level, appear as the combination of instruction with practice and body pattern; in the theoretical level, it is about the culture appeal of martial arts explanation.

\section{A. Return to the Traditional Combination of Training and Practice}

The arrangement and combination of martial arts' movements are the direct expression of the primitive essence of martial arts culture, reflecting the movement variation of attack and defense. In order to complete the task of martial arts teaching, under normal circumstances, we generally require teaching different martial arts routines each semester in accordance with teaching syllabus. Each movement in martial art routine has attack or defense tactical awareness. In order to make students feel the charm of martial arts more deeply, we combine instruction with practice. It is a more traditional teaching method and concept, which is close to the nature of martial arts.

In the combination of instruction with practice of martial arts teaching, "instruction" represents the complete study of martial arts routine movement, "practice" represents single movement of routine, we put it into pure combat awareness after explaining the use of attack or defense. For example, the "cover fist, then raise knee and thread palm" in "Five-step 
boxing" routines requires to turn around when covering fist, with large movement range; when raising knee, keep toes pointed and knee joint higher than the waist; when threading palm, keep arm straight. If combining the movement with the single movement of "Yaozirulin” in Xinyi Boxing, we should use the subjective feeling of the body to feel the attacking way of thrust palm with defense and attack meaning through the similarities between the two movements. So that students can easily build the image of martial arts attack and defense when practicing the routine, imaging an attack scenario to help them better grasp the point of force of covering fist and threading palm.

In modern society, although martial arts routines pay more attention to the value of entertainment and performance, when integrating the actual "fight" into the martial arts teaching, it will help students better understand and master the strength performance of martial arts movements, fully adhering to the essential attribute of martial art culture and developing the uniqueness of martial art teaching.

\section{B. The Action Set of Bodily Details}

Complete martial arts routine is composed of many separate martial arts movements. In the rhythm arrangement of routine practice, it is divided into connection movement and fixed movement. Fixed movement is generally fixed in a certain movement. First is to control practice rhythm and adjust the breathing. Second is to fully show the spirit and temperament of martial arts practitioners in the particular moments. The choice of martial arts set, at the beginning of routine creation, is very focused on the stretch degree of joint stretch. Generally, the main four bodily details include shoulder joint, hip joint, elbow joint and knee joint. Therefore, the fixed movement as a single movement which is kept for a long time in the competition process must strictly require the quality of movement. Teaching process is also a process to sculpture limb details, and a very important teaching link in martial arts teaching.

Martial arts set has very strict requirements and norms for the control of body and details. The basic point of judgment and teaching is whether the fixed movement has martial art's characteristics; whether the joints are stretched; whether the eyes and temperament are dignified and so on. Among them, the use of the eyes is the unique Jing-Qi-Shen expression of martial arts. Under normal circumstances, the eyes need to match up the attack actions. The eyes are basically required to look to the attack direction. But it needs to be emphasized that the eyes can't focus on the action, but the extension of action, that is to look at the distant point in the attack direction.

The pursuit of action standards in sports, in fact, is eager to develop a habit of action standard. The martial arts covers the martial arts movements and daily physical exercise through the support of joints stretching, temperament improvement and other normal actions.

\section{The Martial Arts Identity of Cultural Appeal}

Cultural appeal is the theoretical teaching content of martial arts education. It is theory explanation teaching from aspects of martial arts history, martial arts allusion, martial arts fist plants, martial arts competition rules, etc. Martial arts theory teaching is martial arts project introduction from pure culture perspective, covering martial arts' tradition and modern , confrontation and exercise, health and fitness, and martial arts cultural aesthetics.

Martial arts culture condenses a large number of traditional Chinese culture connotations, and expresses the Chinese philosophy, traditional Chinese medicine theory and other traditional knowledge system in a physical way. For example, the push palm of tai chi requires that the elbow joint of the arm can't be fully stretched and maintain a rounded and harmonious appearance of the body. Through associating with the "beyond is as wrong as falling short" emphasized by the traditional culture, it embodies the ancient Chinese philosophical idea of "things will develop in opposite direction when they become extreme", so as to educate students to leave adequate leeway when doing things and conduct comprehensive analysis when considering things.

Based on this, with the change of society, as the symbolic ideology of Chinese traditional culture, martial arts extends the cultural inheritance signifier of body memory from different levels, and cultivates martial arts practitioners' sense of identity with traditional culture.

\section{The Cultivation of ReCessive Aesthetics in Martial ARTS ROUTINE TEACHING}

In college martial arts teaching, the teaching and exercise of external technical movement is the basic requirements of teaching tasks. Martial arts education process should not only stay in the communication of movements and completion of the syllabus. Teachers should take the initiative to extract the internal conservation under the presentation of martial arts routine, explore the aesthetic idea and taste of knowing and appreciating beauty through the movements of human body.

\section{A. The Strength Beauty of Martial Arts}

Strength is a self-contained property of martial arts. It is a subtle joint reaction between muscle and strength. Martial arts movement without strength can't meet audience and practitioners' expectation for martial arts cultural speculation. The strength expression of martial arts needs to use the "fight" in the single-type exercises and add the imaginary schema of "attack and defend", so as to restore the biological instincts of the human body and enhance the force of martial arts logic. Then let the single-type exercise return to routine practice eventually. Although the strength of martial arts is shown as an abstract picture, the imaginary schema that body interlocutor obtains includes the aesthetic feeling of martial arts charm and style.

The strength beauty of martial arts comprehensively shows the power beauty and artistic beauty of human body, "do not use too much power when making movements, but maintain the fluency of movement. The strength can only be developed when the point of strength is correctly found."[4] In traditional martial arts practice, there is a proverb "develop strength through breath", which means let the adjustment of breath help complete the power generation of martial arts movement. The breath adjustment of martial arts includes "raise, hold, gather, 
sink". Different adjustment methods can lead to the spatial alternation of direction and speed in martial arts movements. The permutation and combination of breath flow constitute the unique charm of martial arts[5]. The strength beauty of martial arts is a body feeling obtained through comprehension. Only through the long-term practice on method can we really comprehend the connotation of strength beauty.

\section{B. The Shape Beauty of Martial Arts}

Shape determines the intuitionistic needs of martial arts exercises. The scholars in our country have studied the morphological characteristics of martial arts athletes. They point out that the body shape is an important quality indicator of martial arts[6]. Martial art's figure beauty belongs to explicit beauty, which combines martial arts' requirements to the movement posture of "hand, eyes, body, law, step", emphasizing correct, harmonious and unified performance intention. In martial arts' set practice, we conduct aesthetic guidance on human body's daily habits through carving movement details, mainly the stretch of shoulder joint and coordination degree of upper and lower limb movements. For example, normally human body's shoulder joints will have three body shapes: chest inward and shrug shoulders, chest out and shoulder straight, chest extension and shoulder backward, giving three different aesthetic feedbacks.

Relatively speaking, the stretch of shoulder joint reflects self-confident, positive, healthy and other psychological feelings. Shrug will give us the first impression of timid, dispirited and other morbid feelings. Based on this, martial arts' training requirements of stretching shoulder can affect or even change the practitioners' shoulders stretch state, thereby forming coordinated and upright shape beauty.

College martial arts teaching content mainly focuses on Changquan and Taijiquan. The routine training style often is stretching posture, symmetrical structure and self-confidence. Each movement detail instruction of teachers can help students maintain shape when practicing martial arts, so as to further extend to the body performance in daily activities and promote the improvement of personal temperament.

\section{The Culture Beauty of Martial Arts}

The culture beauty of martial arts is the need of martial art culture identity. Enhance national pride and cultural identity through the appreciation of culture, further enhancing students' interest in martial arts. Martial arts culture is an important component of Chinese traditional culture. In a certain sense, the connotation of martial arts culture reflects the classical logic and cognition of Chinese history. To cultivate the cultural beauty of martial arts, it is necessary to have a certain accumulation of traditional culture, know martial arts boxing school and understand the philosophical basis of different boxing. In terms of the classification of martial arts boxing school, we need to understand the different cultural history, and treat the connotation of martial arts movement from the cultural perspective, rather than generally treating martial arts as "fighting" technique.

The cultivation of martial arts culture beauty helps students correctly understand the significance of martial arts. In fact, in contemporary society, most of martial arts culture represents a kind of life thinking and tenacity spirit. The cultivation of martial arts culture beauty can effectively reduce the problem of "use martial arts to beat people after learning it" and other similar misunderstanding problems,

\section{CONCLUSION}

Martial arts aesthetic education has certain recessive cultivation characteristics, because it depends on the development of martial arts teaching consciousness. Therefore, to improve the aesthetics of martial arts, we must consider the effectiveness of martial arts teaching methods, find teaching methods and training content with penetration function and martial arts significance. College martial arts teaching needs to combine with the cultural characteristics of martial arts. Martial arts teaching is not simply the communication of martial arts movements. It needs to take advantage of body exercises of martial arts to let students realize the martial arts beauty of life principle, self-discipline, tenacity and harmony that martial arts pursues intrinsically.

\section{REFERENCES}

[1] Wang Gang, Wu Song. Chinese martial arts: cultural belonging of traditional aesthetic[J]. Journal of Sports Culture .2007.5: 23-28(In Chinese)

[2] Marcel Mauss, translated by She Biping. Sociology and anthropology[M]. Shanghai: Shanghai Translation Publishing House .2003: 301. (In Chinese)

[3] Zhao Fuxue, Cheng Chuanyin. Study on the relationship between coercion and freedom in school physical education [J]. Journal of Sports Science. 2016.3 (36): 89-94(In Chinese)

[4] Cui Yuqiang. Aesthetic characteristics and artistic study of martial arts routine movements[J]. Contemporary Sports Technology. 2015.31 (5): 249-251(In Chinese)

[5] Qin Hua. Aesthetic education of martial arts teaching in colleges and universities [J]. Journal of Weinan Teachers College. 2014.4 (29): 2932(In Chinese)

[6] Ma Lingjuan. Body shape and quality characteristics of China's elite women gun and sword athletes[D]. Beijing Sport University.2014: 2427(In Chinese) 